\title{
Intelligent interactions for multimedia processing: an editorial
}

\author{
Jason J. Jung
}

Published online: 12 September 2012

(C) Springer Science+Business Media, LLC 2012

Recently, the research fields of social interactions among people have received a great deal of attention in recent years and, beyond doubt, have established themselves as main cornerstones of intelligent multimedia processing. Learning and extracting patterns from multimedia data is inseparably connected with various types of uncertainty. Yet, it is becoming more and more apparent and accepted among researchers that collaborative interactions between people can be applied to various potential solutions $[1,2]$.

In this special issue, we are focusing on recent advances in the use of social interaction methods for multimedia processing. We especially welcome contributions on the use of formal frameworks for reasoning under integrating human intelligence, such as collaborative taggings, social mining, and machine learning, including but not limited to data preprocessing, model induction (e.g., classification, regression).

The paper "Agent-mediated shared conceptualizations in tagging services", authored by Aranda-Corral et al., deal with the problem on tagging behaviors of different users which causes semantic heterogeneity in tagging systems. Thereby, an agent-based reconciliation knowledge system based on Formal Concept Analysis (FCA), has been applied to facilitate the semantic interoperability between personomies (personal folksonomies).

The paper "Emotion-based character clustering for managing story-based contents: a cinemetric analysis”, authored by Jung et al., has proposed an interesting method that can clusters entities by measuring emotional similarities between the entities. Particularly, this work introduces a cinematic approach to process movie contents.

The paper "Semantics enhanced engineering and model reasoning for control application development”, authored by Hästbacka and Kuikka, claims that an approach applying Web Ontology Language (OWL) semantics and reasoning to models is presented with examples to support industrial control application engineering.

J. J. Jung $(\bowtie)$

Department of Computer Engineering, Yeungnam University, Gyeongsan, Korea

e-mail: j2jung@gmail.com 
Moreover, the study proposes the generalized classifications can be inferred from instance models and combined with generic engineering knowledge maintained in ontologies.

The paper "Relational large scale multi-label classification method for video categorization", authored by Indyk et al., is focusing on dealing with a problem on automated video categorization in large datasets. The study has proposed an interesting Iterative Multi-label Propagation (IMP) algorithm for relational learning in multi-label data.

The paper "A term normalization method for efficient knowledge acquisition through text processing", authored by Hwang et al., emphasizes the importance of knowledge management on 'Big' Data. Thereby, term extraction and normalization is regarded as the most serious step for doing that. The study has proposed a method of term normalization which finds a normalized form (original and standard form defined in dictionaries) of variant terms.

The paper "Tuning user profiles based on analyzing dynamic preference in document retrieval systems", authored by Mianowska and Nguyen, claims that user profiling is an important step for personalized information retrieval systems. Thereby, this work has designed a model for user profile tuning in document retrieval systems. An interesting method for tuning the user profile based on analysis of user preferences dynamics has been experimentally evaluated to confirm whether with growing history of user activity the created user profile can converge to his preferences.

The paper "Preference-based user rating correction process for interactive recommendation systems", authored by Pham and Jung, has assumed that there are mistakes on user ratings, and it makes the recommendation performance decreased. In this paper, authors have focused on an interactive recommendation system which can help users to correct their own ratings. Thereby, they have proposed an interesting method to determine whether the ratings from users are consistent to their own preferences (represented as a set of dominant attribute values) or not and eventually to correct these ratings to improve recommendation.

The paper "Axiomatic characterization of belief merging by negotiation", authored by Tran et al., has claimed that belief merging has been an active research field with many important applications. Especially, various approaches on belief merging require that all belief bases are provided explicitly and the role of agents, who provide the belief bases, are not adequately considered In this study, authors have proposed the merging problems from other point of view. Namely, they treat a belief merging problem as a game, in which rational agents participate in a negotiation process to find out a jointly consistent consensus trying to preserve as many important original beliefs as possible.

The paper "Detecting and tracking of multiple pedestrians using motion, color information and the AdaBoost algorithm", authored by Lim and Kim, has focused on robust detection and tracking of pedestrians in image sequences are essential for many vision applications. Thereby, authors have proposed a method to detect and track multiple pedestrians using motion, color information and the AdaBoost algorithm. Particularly, this approach has detected pedestrians in a walking pose from a single camera on a mobile or stationary system.

This special issue has been achieved by a number of fruitful collaborations. We would like to thank the editor in chief of Multimedia Tools and Applications 
(MTAP), Prof. Borko Furht, for his kind support and help during the entire process of publication. The special issue has selected 9 high-quality papers out of 27 submissions (about $33.3 \%$ acceptance rate). This was possible thanks to the work of the renowned researchers that provided their anonymous reviews.

Finally, we are most grateful to the authors for their valuable contributions and for their willingness and efforts to improve their papers in accordance with the reviewers suggestions and comments.

\section{References}

1. Jung JJ (2011) Boosting social collaborations based on contextual synchronization: an empirical study. Expert Syst Appl 38(5):4809-4815

2. Tian YH, Srivastava J, Huang T, Contractor NS (2010) Social multimedia computing. IEEE Computer 43(8):27-36

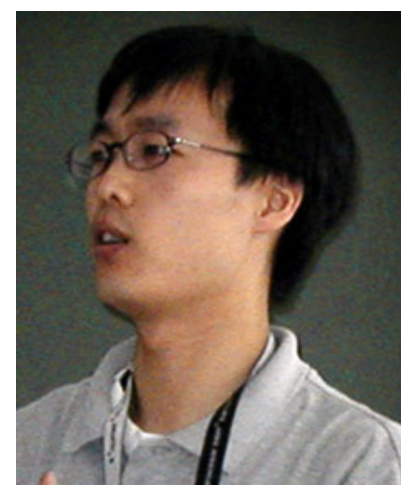

Jason J. Jung is an assistant professor of Computer Engineering Department at Yeungnam University, Korea. He was a postdoctoral researcher in INRIA Rhone-Alpes, France in 2006, and a visiting scientist in Fraunhofer Institute (FIRST) in Berlin, Germany in 2004. He received the B.Eng. in Computer Science and Mechanical Engineering from Inha University in 1999. He received M.S. and Ph.D. degrees in Computer and Information Engineering from Inha University in 2002 and 2005, respectively. His research topics are knowledge engineering on social networks by using machine learning, semantic Web mining, and ambient intelligence. He has about 25 international journal articles published in KnowledgeBased Systems, Information Retrieval, Information Processing \& Management, Knowledge and Information Systems, and Expert Systems with Applications. Also, he is an editorial member of Journal of Universal Computer Science and International Journal of Intelligent Information and Database Systems. Moreover, he has been editing 10 special issues in Information Sciences, Journal of Network and Computer Applications, Computing and Informatics and so on. 